\title{
Evolution of the excitonic state of DNA stacked
}

\author{
thymines: intra-base $\pi \pi^{*} \rightarrow \mathrm{S}_{0}$ decay paths account
}

for ultrafast (sub-ps) and longer ( $>100 \mathrm{ps})$

\section{deactivations}

Irene Conti ${ }^{\dagger}, *$ and Marco Garavelli $i^{\dagger, *}$

† Dipartimento di Chimica Industriale “Toso Montanari”, Università di Bologna, Viale del

Risorgimento 4, I-40136 Bologna, Italy

AUTHOR INFORMATION

Corresponding Author

E-mail: irene.conti@unibo.it

E-mail: marco.garavelli@unibo.it.

ABSTRACT. Monomer-like ring puckering decay paths for two stacked Quantum Mechanical Thymines inside a solvated DNA duplex described at the molecular mechanics level are mapped using a hybrid CASPT2//CASSCF/MM protocol that accounts for steric, electronic and electrostatic interactions within the nucleobases native environment. Asymmetric stacking 
between nucleobases open ups different intra-base $\pi \pi^{*}$ decay paths accounting for distinctive excited state lifetimes, spanning the sub-ps to sub-ns time window.

\section{TOC GRAPHICS}

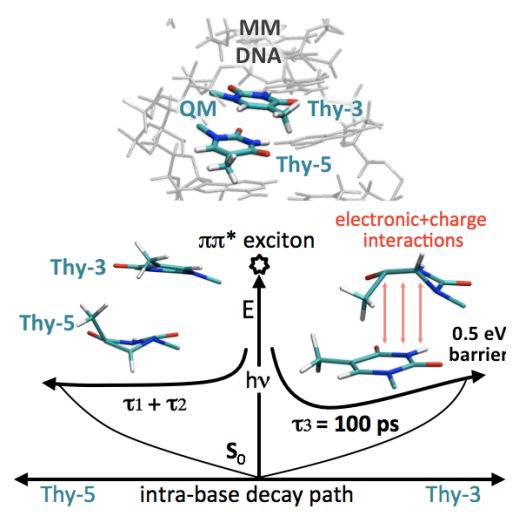

KEYWORDS. Stacked thymines, exciton state, monomer-like, deactivation path, lifetimes, puckering.

Nucleobases, nucleosides, nucleotides and, progressively increasing the complexity, multimers and single/double strand DNA/RNA show different photophysical behaviours arising by the emerging collective properties of the multichromophoric system..$^{1-4}$ For instance, a relevant feature of nucleobase multimers is an excited state lifetimes spanning a wider range of time scales $^{7-11}$ : in addition to the ultrafast decay times $\left(\tau_{1}\right.$ and $\left.\tau_{2}\right)$ observed already in isolated nucleobases and the monomers, in the order of hundreds femtseconds (fs) to few picoseconds (ps) and assigned to direct decay to the ground state of a localized $\pi \pi^{*}$ excitation $^{5,12,14-18}$ a longer lifetime component $\tau_{3}$, in the order of tens to hundreds ps, emerges or is reinforced. The reasons behind this behaviour are still strongly debated, the formation of a longer living intra/inter-strand charge transfer (CT) state between two stacked/paired nucleobases being the most credited 


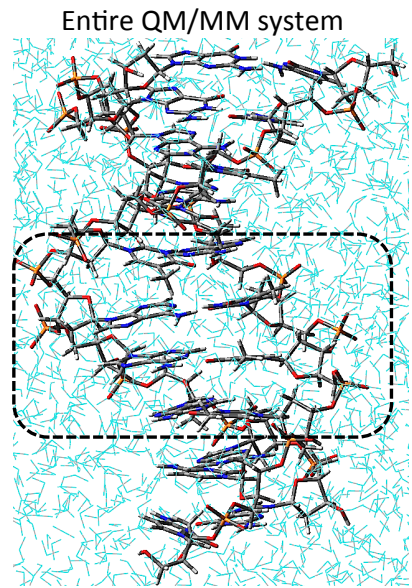

Scheme 1

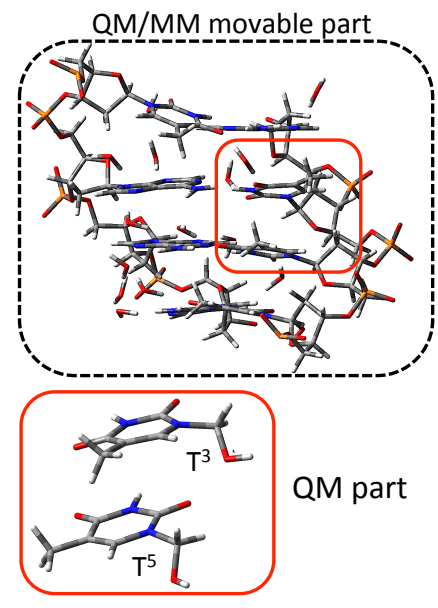

hypothesis $^{13,20-23}$. Pyrimidines, however, display the longer component already at the monomer level, a feature that has been assigned to the population of a dark $n \pi^{*}$ state, calling also for an intra-base mechanism as a possible explanation for the longer $\tau_{3}$ component when pyrimidines are present ${ }^{12,24}$. Accurate simulations and computational protocols which allow to study larger systems, like the hybrid QM/MM approaches, represent the elective tools to account for experimental observations and deliver the molecular movie behind the recorded signals. In this direction, we developed, and here we employ, a hybrid quantum mechanics/molecular mechanics (QM/MM) scheme ${ }^{12,28}$ that couples state-of-the-art ab initio multi reference dynamically correlated methods (CASPT2//CASSCF) of the photoactive moiety, i.e. the photoexcited nucleobases, with an explicit classical atomistic model (Amber force field) of the environment, i.e. the embedding DNA duplex and the solvent (see Scheme 1).

In the present Letter, we analyse how the classical monomer-like $\pi \pi^{*} \rightarrow \mathrm{S}_{0}$ decay paths (defined as 'ethene-like') of two stacked Thymines (Thys), and responsible for the ultrafast decays of the single solvated nucleobase ${ }^{4-6,25}$, are affected by the DNA environment leading to much longer excited state lifetimes. For this purpose, we employ the solvated double strand $B$-fragment 5'-CC-T-[T $\left.{ }^{5}-\mathrm{T}^{3}\right]-\mathrm{A}-\mathrm{A}-\mathrm{A}-\mathrm{G}-\mathrm{G}-3$ ' $\left(1 \mathrm{ikk} \cdot \mathrm{pdb}^{30}\right)$, where $\mathrm{T}^{5}$ and $\mathrm{T}^{3}$ are the QM Thys at the 5'-end and 3'end position, respectively. Scheme 1 shows the QM (red square) and MM partition, indicating the movable MM portion (dashed square) in the QM(CASSCF)/MM(AMBER) optimization scheme employed here (validation of the QM/MM partitioning in the Supporting Information 
$\left.(\mathrm{SI})^{23,31-32}\right)$. Eventually, single point CASPT2//CASSCF $(16,14) / \mathrm{MM}$ calculations were performed to deliver realistic energies of the optimized critical points and Conical Intersections (CIs) (see the Computational Methods in the SI for more details).

Recent studies ${ }^{25,29,33}$ reveal that exciton states delocalized over just two nucleobases represent about half of the lowest UV absorption band. Thus, despite the fact that initial excitation can also produce an exciton delocalized over a larger number of stacked nucleobases, initial excitation and localization of the excitation energy into two Thy, as well as the resulting photoinduced decay, is expected to be well described by the current approximation that represents a suitable excitonic model for a couple of stacked Thys within either homo- or hetero-DNA multimers. In conclusion, while the present work cannot consider larger excitonic states as well as feasible base-pair CT states (and their related electron-proton transfer decay path) and do not contemplate intra-strand CT processes between adjacent bases (even if the present QM/MM model should be large enough), it is fully catching the photophysics behind a dimeric exciton of two stacked Thys (that is the major component in the excitonic description ${ }^{25,29,33}$ ) and the successive fundamental localization process followed by the monomer-like evolution of the excitation energy, in its native environment.

Generally speaking, when considering intra-base 'ethene-like' $\pi \pi^{*} \rightarrow \mathrm{S}_{0}$ decay paths of a couple of stacked Thys, four different (non equivalent) channels emerges due to the DNA asymmetric environment: two different ring puckering are possible for each base, recognisable by the subsequent methyl out of plane bending, towards the 5'-end or the 3'-end, respectively. 

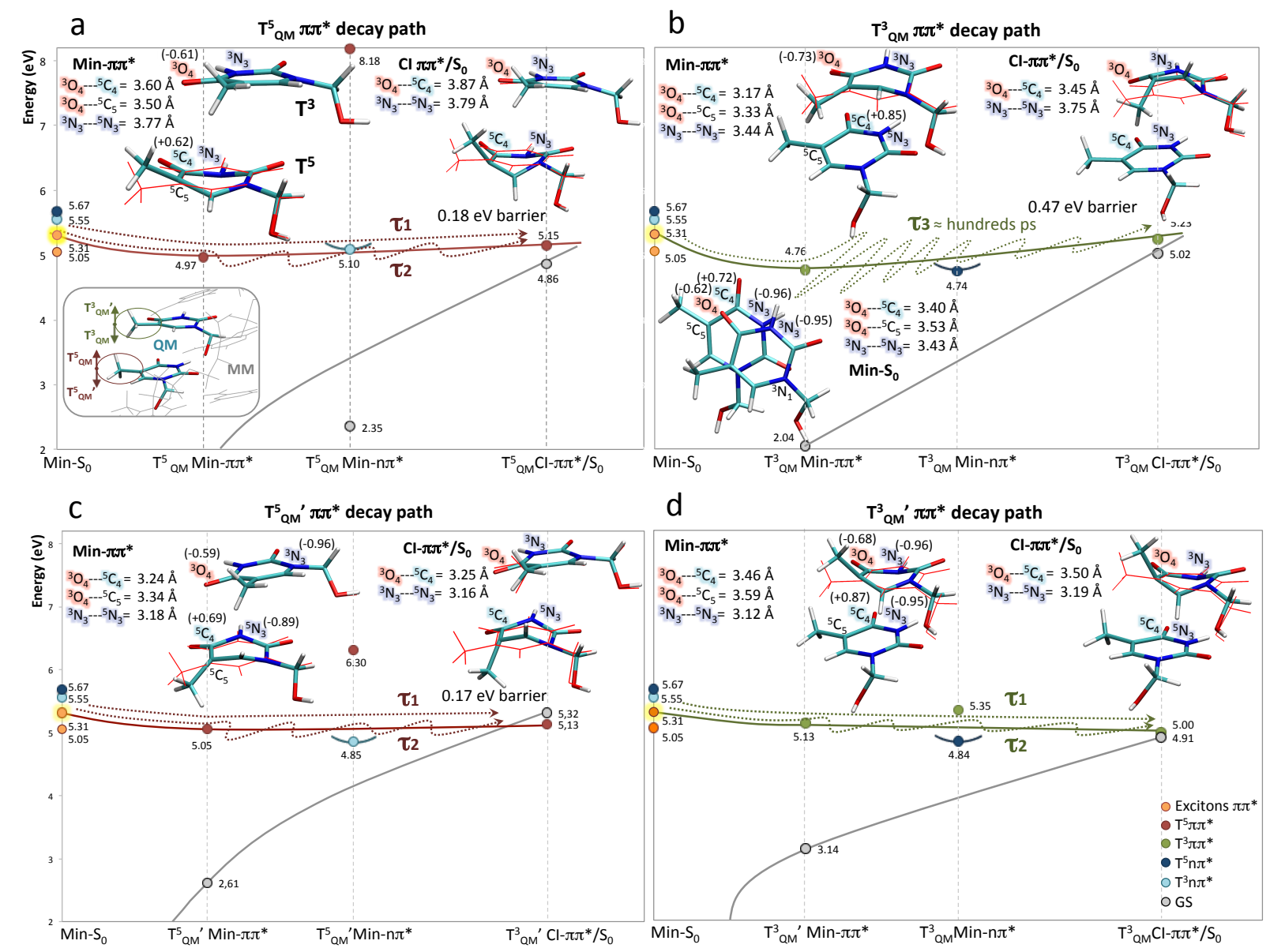

Figure 1. $\pi \pi^{*}$ monomer-like decay paths of two stacked Thys inside DNA double strand. They are characterized by four different methyl motions, clearly explained in the bottom-left of figure 1a, that are $\mathrm{T}_{\mathrm{QM}}^{5}(\mathrm{a}), \mathrm{T}^{3}{ }_{\mathrm{QM}}(\mathrm{b}), \mathrm{T}_{\mathrm{QM}}^{5}$ (c) and $\mathrm{T}^{3}{ }_{\mathrm{QM}}$ ' (d). Minima and CIs (Min- $\pi \pi^{*}$ and $\mathrm{CI}-\pi \pi^{*} / \mathrm{S}_{0}$ ) are overlapped to Min- $\mathrm{S}_{0}$ red structure, to better understand the $\mathrm{T}^{5}-\mathrm{T}^{3}$ motions along the paths. Dotted lines represent the different kind of decay paths associated to $\tau_{1}, \tau_{2}$ or $\tau_{3}$, respectively, in each panel (a, b, c and d). n $\pi^{*}$ minima (Min-n $\left.\pi^{*}\right)$ structures are shown and explained in SI section, Scheme S4.

In the current work, these four paths are exemplified in the bottom-left square of Figure 1a, and labelled $\mathrm{T}_{\mathrm{QM}}^{3}$ or $\mathrm{T}_{\mathrm{QM}}^{3}$, and $\mathrm{T}_{\mathrm{QM}}^{5}$ or $\mathrm{T}_{\mathrm{QM}}^{5}$. Mapping all these mechanisms within the current

QM/MM model, the whole inter-base $\mathrm{T}^{5}---\mathrm{T}^{3}$ electronic/steric interactions, that are a

fundamental component in diversifying the photoinduced dynamics evolution of a Thy-Thy dimeric exciton (as compared to single solvated nucleobases) are fully accounted for at the QM level.

Vertical excited states computed out of the Ground State (GS) minimum (Min- $\mathrm{S}_{0}$ ) of the B-DNA multimer reveal two $\pi \pi^{*}$ states absorbing at 5.05 and $5.31 \mathrm{eV}$, respectively, the highest one 

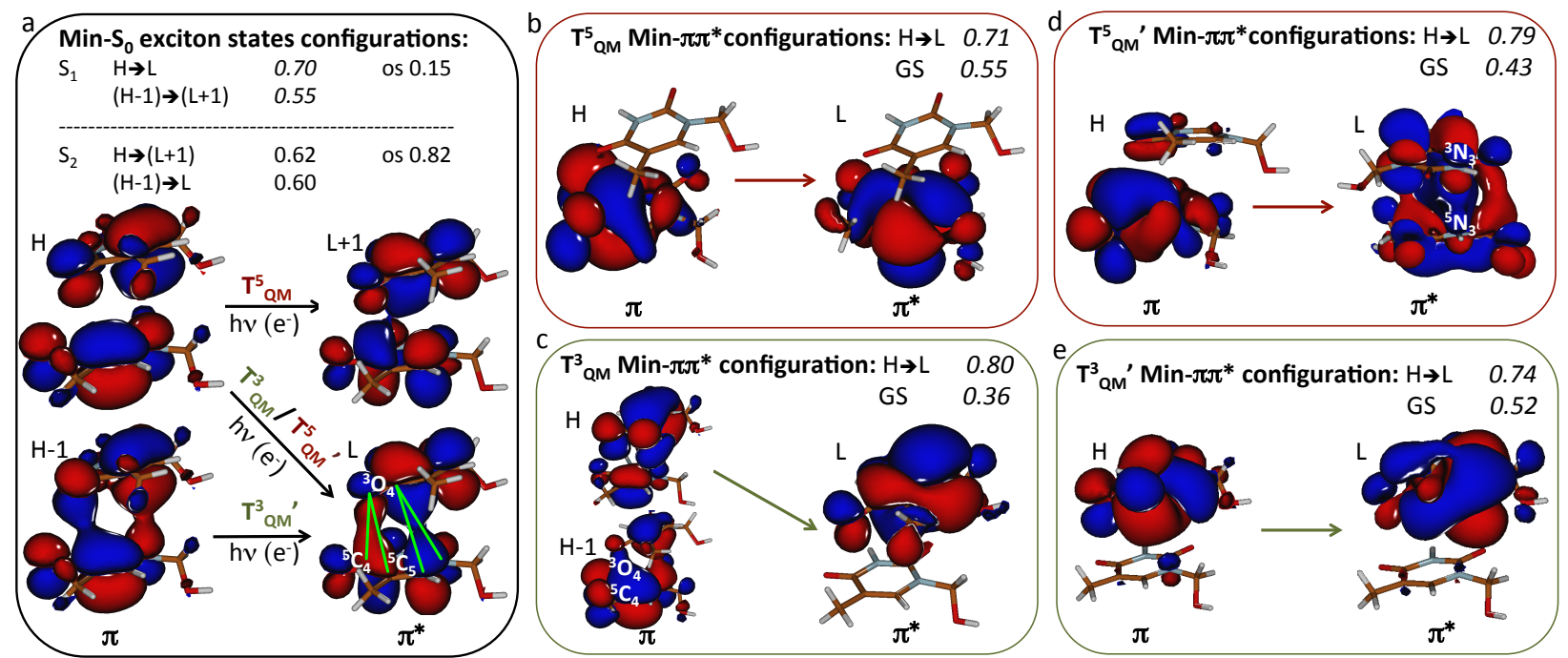

Figure 2. Molecular orbitals (Homo (H), Homo -1 (H-1), Lumo (L), and Lumo+1 (L+1)) involved in the electronic transitions discussed in the text for (a) Min- $\mathrm{S}_{0}$ and (b), (c), (d), (e), the four $\pi \pi^{*}$ mimima, $\mathrm{T}_{\mathrm{QM}}^{5}$ Min- $\pi \pi^{*}, \mathrm{~T}_{\mathrm{QM}}^{3}$ Min- $\pi \pi^{*}, \mathrm{~T}^{5}{ }_{\mathrm{QM}}$ ' Min- $\pi \pi^{*}$ and $\mathrm{T}_{\mathrm{QM}}^{3}$ ' Min- $\pi \pi^{*}$, respectively Oscillator strengths (os) and dominant CASSCF wavefunction coefficients are also documented. A larger number of orbitals is shown in the SI.

showing the larger oscillator strength (see $\mathrm{Min}_{-} \mathrm{S}_{0}$ in Figure $2 \mathrm{a}$ and Table S2 in SI section). They both show a strong exciton nature, with the excitation which is indeed highly delocalized on the two bases, as the $\pi$ and $\pi^{*}$ orbitals (completely diffused on both bases) and the excited state configurations (presenting similar coefficients in both states) show (see Figure 2a). The solvatochromic blue-shift of the $n \pi^{*}$ states observed in a polar environment is also well reproduced $^{4,27,34}$.

Relaxation of the bright $\pi \pi^{*}$ state leads to an $S_{1}$ state where the excitation energy has been localized either on $\mathrm{T}^{5}$ or $\mathrm{T}^{3}$, thus triggering intra-base decay paths as described below. However, and remarkably, the energy profiles and the ring distortions along the four possible 'ethene-like' decay paths (Figure 1a, b, c and d) show significant quantitative and qualitative differences. This is due to asymmetric $\mathrm{T}^{3}-\mathrm{T}^{5}$ stacking orientation, leading to different monomer-like decay paths, arisen from non-equivalent electronic, electrostatic and sterical interactions. In the same way, different stacking ways should give, for the same reason, additional modified decay paths, as recent publications demonstrate ${ }^{35-36}$. 
The upper part of Figure 1 shows $\mathrm{T}^{5}$ and $\mathrm{T}^{3}$ ring puckerings leading the out of plane methyl motion vs. $\mathrm{T}^{3}$ and $\mathrm{T}^{5}$ respectively: they are called $\mathrm{T}^{5}{ }_{\mathrm{QM}}$ and $\mathrm{T}_{\mathrm{QM}}^{3}$ mechanisms, shown in Figure 1a and $1 \mathrm{~b}$ respectively. The opposite methyl motions $\left(\mathrm{T}_{\mathrm{QM}}^{5}\right.$, and $\mathrm{T}_{\mathrm{QM}}^{3}$, Figure $1 \mathrm{c}$ and $\mathrm{d}$ respectively), move the bases a bit closer. The square at the bottom-left of Figure 1a, mentioned above, clearly addresses the methyl movements to the corresponding labels.

The $\mathrm{T}_{\mathrm{QM}}^{5}$ mechanism and energetics match the one observed in solvated nucleobases ${ }^{5-6}$, i.e. it behaves like an isolated base, being only slightly affected by the DNA environment. Namely, an extended excited state energy plateau characterizes the ring-puckering/methyl-bending reaction coordinate that drives the system into the decay funnel (CI- $\pi \pi^{*} / \mathrm{S}_{0}$, Figure 1a) with a small barrier $(\sim 0.18 \mathrm{eV})$, as also found for the nucleoside in water $\left(\sim 0.1 \mathrm{eV}\right.$ at the CASPT2/MM level ${ }^{5}$ and $\sim 0.2 \mathrm{eV}$ at the TD-DFT/PCM level $\left.{ }^{6}\right)$. The $\mathrm{T}_{\mathrm{QM}}^{5} \pi \pi^{*}$ minimum $\left(\mathrm{T}^{5}{ }_{\mathrm{QM}}\right.$ Min- $\left.\pi \pi^{*}\right)$ shows a 'boat-like' ring deformation, matching to the ring distortion observed in all solvated pyrimidines $^{5-6}$ (see THD, URD and CYD Min- $\pi \pi^{*}$ in Table 1 ). The somehow slightly smaller methyl bending in the $\mathrm{T}^{5}{ }_{\mathrm{QM}} \mathrm{CI}$ region (see CI- $\pi \pi^{*} / \mathrm{S}_{0}$ in Table 1) is due to the DNA steric constraints that are, however, not so demanding to change (both qualitatively and quantitatively) the mechanism observed in the isolated nucleoside. This is also revealed by the $\pi(\mathrm{H})$ and $\pi^{*}$ $(\mathrm{L}+1)$ orbitals (see Figure 2a) involved in the description of the excitation leading to the $\mathrm{T}_{\mathrm{QM}}^{5}$ monomer-like distortion, that indeed display mainly anti-bonding inter-base character and, thus, an electronic nature that very much resembles the one found in the solvated thymidine ${ }^{5}$.

This decay path is expected to lead to ultrafast excited states life times resembling the ones observed in the single base, namely $\tau_{1}$ and $\tau_{2}$ (see 'ultrafast life times' in Table 2), that thus persist in stacked multimeric systems (such as poly(A)·(T) or poly(T)) as indeed observed. $\tau_{1}$ 
Table 1. $\pi \pi^{*}$ minima and $\pi \pi^{*} / \mathrm{S}_{0} \mathrm{CI}$ structures belonging to monomerlike paths of $\mathrm{T}_{\mathrm{QM}}^{5}, \mathrm{~T}^{3}{ }_{\mathrm{QM}} \pi \pi^{*}$ decay routes found in DNA and in solvated pyrimidines (THD, URD and CYD) $)^{5}$. Torsional parameters values (A, B, $\mathrm{C}, \mathrm{D}, \mathrm{E}, \mathrm{F}, \mathrm{g}$ ) labeled in the top picture are documented in Table S1 (SI). $\mathrm{T}^{5}{ }_{\mathrm{QM}}$ ' and $\mathrm{T}^{3}{ }_{\mathrm{QM}}$ ' minima (Min- $\left.\pi \pi^{*}\right)$ and $\mathrm{CIs}\left(\mathrm{CI}-\pi \pi^{*} / \mathrm{S}_{0}\right)$ structures are largely resembling $\mathrm{T}^{5}{ }_{\mathrm{QM}} \mathrm{Min}-\pi \pi^{*}$ and $\mathrm{CI}-\pi \pi^{*} / \mathrm{S}_{0}$ structures, see Scheme S3..

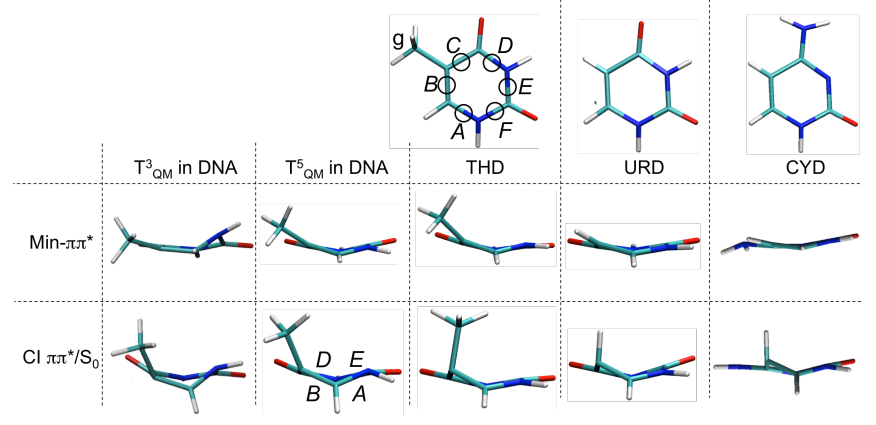

could be associated either with the initial relaxation of the wave packet out of the Franck-Condon (FC) region, until reaching the $S_{1}$ energy plateau, or to direct ballistic decay of part of the excited state population when starting from favourable vibrational levels directly driving the system into the CI- $\pi \pi^{*} / \mathrm{S}_{0} . \tau_{2}$ should instead account for part of the excited state population wondering around the flat energy region before overcoming the small barrier $(0.18 \mathrm{eV}$, Figure 1a) to reach the funnel and decay to the ground state s-6,37 $^{4}$

On the other hand, the $\mathrm{T}_{\mathrm{QM}}^{3}$ intra-base decay path (showing the largest starting gradient, and therefore energetically favourite, see Figure 1b), in which methyl bends toward the adjacent QM base $\mathrm{T}^{5}$ (Figure $1 \mathrm{~b}$ ), shows a qualitatively different molecular distortion and energy profile with a more stable $\pi \pi^{*}$ minimum $\left(\mathrm{T}^{3}{ }_{\mathrm{QM}}\right.$ Min- $\left.\pi \pi^{*}\right)$, differentiating itself from the (above described) classical monomer-like decay path ${ }^{5,24}$. The electronic reason behind this behaviour, that is also responsible for the qualitative difference between the two monomer-like paths, can be understood when looking at the $\pi$ and $\pi^{*}$ orbitals involved in the description of the two localized excited state minima and paths (see Figure $2 \mathrm{a}$ ). $\mathrm{T}^{5}{ }_{\mathrm{QM}}$ decay path, as mentioned above, involves $\pi$ and $\pi^{*}$ orbitals ( $\mathrm{H}$ and $\mathrm{L}+1$, respectively) with inter-base anti-bonding character, thus leading to weak $\mathrm{T}^{3}-\mathrm{T}^{5}$ electronic interactions and to a very localized excited state (see $\mathrm{H}$ and $\mathrm{L}$ in $\mathrm{T}^{5}{ }_{\mathrm{QM}}$ Min$\pi \pi^{*}$, Fig. 2b), closely resembling the one in the free solvated nucleoside. On the contrary, the Min- $\mathrm{S}_{0} \pi^{*}(\mathrm{~L})$ orbital involved in the $\mathrm{T}^{3}{ }_{\mathrm{QM}}$ monomer-like decay shows an inter-base bonding 
nature (see the green lines in Figure 2a), enforcing a stronger $\pi$-stacking between the two Thys, that get them close and lead to a more stable minimum: the driving force leading to the $\mathrm{T}_{\mathrm{QM}}^{3}$ Min- $\pi \pi^{*}$, indeed, reduces the corresponding inter-base distances, as exemplified by the shortening in the ${ }^{3} \mathrm{O}_{4^{---}}{ }^{5} \mathrm{C}_{4}$ and ${ }^{3} \mathrm{O}_{4^{---}} \mathrm{C}_{5}$ distances (from $3.40 \AA$ to $3.17 \AA$ and from $3.53 \AA$ to $3.33 \AA$, respectively, see Figure $1 \mathrm{~b})$, along the inter-base $\pi^{*}(\mathrm{~L})$ bonding orbitals. Moreover, the non-negligible GS contribution on the $S_{1}\left(\pi \pi^{*}\right) T^{3}{ }_{\mathrm{QM}}$ Min- $\pi \pi^{*}$ wave function (see Figure 2c) further contributes to enforce the $\pi$-stacking inter-base bonding interaction (due to the $\pi$ orbitals diffuses on both Thys, see also Scheme $\mathrm{S} 1$ and $\mathrm{S} 2 \mathrm{~b}$ in the SI), a feature that is loosed in $\mathrm{T}_{\mathrm{QM}}^{5}$ Min- $\pi \pi^{*}$, where the orbitals are completely localized (see $\pi$ orbitals Figure $2 b$ and Scheme S2a).

This kind of interaction tends to preserve roughly the base-base equilibrium distance found in the GS minimum, and to keep the planarity of the system, which is necessary in the $\pi-\pi$ interaction. This is indeed observed in the $\mathrm{T}^{3}{ }_{\mathrm{QM}}$ Min- $\pi \pi^{*}$ (even is some inter-base atoms distance are a bit shortened, see above) where the two staked Thys stay approximately at the same equilibrium distance (due to $\pi$-interaction) as in $\operatorname{Min}-\mathrm{S}_{0}\left(\sim 3.5 \AA\right.$, see the overlap between the Min $\pi \pi^{*}$ and Min- $\mathrm{S}_{0}$ structures (in red) in Figure $1 \mathrm{~b}$ ) and the $\mathrm{T}^{3}{ }_{\mathrm{QM}}$ ring is less distorted compared to $\mathrm{T}^{5}{ }_{\mathrm{QM}}$ Min$\pi \pi^{*}$ : it shows a 'chair-like' structure (just ${ }^{3} \mathrm{C}_{6}$ is moving out of plane, Table 1 ) instead of the 'boat-like' distortion observed in all the solvated pyrimidines $\pi \pi^{*}$ minima ${ }^{5}$ as well as in the $\mathrm{T}^{5}{ }_{\mathrm{QM}}$ case. This is further shown in Table S1, documenting the torsional parameters of the pyrimidine ring in the two $\pi \pi^{*}$ minima (see also the different trend shown by the red line in the bottom insert of Table $\mathrm{S} 1$ ). In the $\mathrm{T}_{\mathrm{QM}}^{5}$ Min- $\pi \pi^{*}$, where the stabilizing $\pi$-stacking is totally lost, the two bases are more distant and largely free to distort the ring ('boat-like', see the $\mathrm{T}_{\mathrm{QM}}^{5} \mathrm{Min}-\pi \pi^{*}$ and Min- $\mathrm{S}_{0}$ overlapped structures (in red) in Figure 1a). 
Table 2. Excited state lifetimes (picoseconds) in solution $\left(\tau_{1}, \tau_{2}, \tau_{3}\right)$ and the amount $(\%)$ of excited molecules that decay via ultrafast $\left(\mathrm{A}_{\mathrm{UF}}\right)$ or slow $\left(\mathrm{A}_{\mathrm{S}}\right)$ channels.

\begin{tabular}{|c|c|c|c|c|c|c|}
\hline & \multicolumn{4}{|c|}{ Ultra-fast life times } & \multicolumn{2}{|c|}{$\begin{array}{l}\text { Tens to hundreds ps } \\
\text { life times }\end{array}$} \\
\hline & $\tau_{1}$ & $\tau_{2}$ & \multicolumn{2}{|c|}{$\mathrm{A}_{\mathrm{UF}}, \%$} & $\tau_{3}$ & $\mathrm{~A}_{\mathrm{s}}, \%$ \\
\hline \multirow{2}{*}{ Thy } & $0.20 \pm 0.02$ a & $0.63 \pm 0.02$ a & \multirow{2}{*}{\multicolumn{2}{|c|}{$-87 \pm 13^{b}$}} & \multirow{2}{*}{$30 \pm 13$ b } & \multirow{2}{*}{$-11 \pm 13 b$} \\
\hline & \multicolumn{2}{|c|}{$2.8 \pm 0.4 \mathrm{~b}$} & & & & \\
\hline \multirow{2}{*}{ TMP } & $0.21 \pm 0.03 \mathrm{c}$ & $1.07 \pm 0.06^{\mathrm{c}}$ & \multirow{2}{*}{\multicolumn{2}{|c|}{$-83 \pm 3 b$}} & \multirow{2}{*}{$127 \pm 15 b$} & \multirow{2}{*}{$-14 \pm 4 b$} \\
\hline & \multicolumn{2}{|c|}{$2.2 \pm 0.1^{\mathrm{b}}$} & & & & \\
\hline $\operatorname{poly}(\mathrm{A}) \cdot(\mathrm{T})$ & $0.5 \pm 0.3 \mathrm{~d}$ & $5.2 \pm 0.5^{\mathrm{d}}$ & $35^{\mathrm{d}}$ & $-43^{d}$ & $98 \pm 30 \mathrm{~d}$ & $-16^{\mathrm{d}}$ \\
\hline $\operatorname{poly}(\mathrm{T})$ & $0.5 \pm 0.2^{\mathrm{d}}$ & $2.2 \pm 0.3^{\mathrm{d}}$ & $36^{\mathrm{d}}$ & $-55^{\mathrm{d}}$ & $102 \pm 30^{d}$ & $-6^{d}$ \\
\hline Ade & $0.81 \pm 0.07^{\mathrm{e}}$ & $2.8 \pm 0.9^{\mathrm{e}}$ & & & & \\
\hline AMP & $0.10 \mathrm{c}, \mathrm{f}$ & $0.52 \pm 0.10^{c}$ & & & & \\
\hline GMP & $0.22^{\mathrm{g}}$ & $0.9^{\mathrm{g}}$ & & & & \\
\hline \multirow{2}{*}{ Ura } & \multicolumn{2}{|c|}{$0.10 \pm 0.03$ a } & \multirow{2}{*}{\multicolumn{2}{|c|}{$-68 \pm 5 b$}} & \multirow{2}{*}{$24 \pm 2 b$} & \multirow{2}{*}{$-28 \pm 5 b$} \\
\hline & & & & & & \\
\hline \multirow{2}{*}{ URD } & \multicolumn{2}{|c|}{$0.21 \pm 0.03 \mathrm{~h}$} & \multirow{2}{*}{\multicolumn{2}{|c|}{$-51 \pm 6 b$}} & \multirow{2}{*}{$147 \pm 7$ b } & \multirow{2}{*}{$-42 \pm 6 b$} \\
\hline & & & & & & \\
\hline Cyt & \multicolumn{2}{|c|}{$2.9 \pm 0.7 \mathrm{~b}$} & \multicolumn{2}{|c|}{$-87 \pm 9 b$} & $12 \pm 3^{b}$ & $-9 \pm 7 b$ \\
\hline \multirow{2}{*}{ CMP } & $0.27 \pm 0.02^{c}$ & $1.38 \pm 0.11^{\mathrm{c}}$ & \multirow{2}{*}{\multicolumn{2}{|c|}{$-56 \pm 34 b$}} & \multirow{2}{*}{$34 \pm 3$ b } & \multirow{2}{*}{$-41 \pm 19 b$} \\
\hline & & & & & & \\
\hline
\end{tabular}

a Excited-state lifetimes measured using femtosecond fluorescence up-conversion (recorded at $330 \mathrm{~nm})^{6}$. ${ }^{\mathrm{b}}$ Femtoseconds transient absorption technique. Ultra-fast lifetimes indicate the complete vibrational cooling. ${ }^{5,12}$ c Time resolved fluorescence spectra $^{5,12}{ }^{\mathrm{d}}$ Transient absorption signals. Vibrational cooling life-times ${ }^{5,7,13}$. ${ }^{\mathrm{e}}$ Transient absorption signals were recorded by the pump-probe technique ${ }^{19-20} .{ }^{\mathrm{f}}$ Time-resolved photoelectron spectroscopy of gas-phase mass-selected nucleotide anions life times: they are not so far from ultrafast lifetimes of solvated nucleotides ${ }^{25-26} .{ }^{g}$ GMP in aqueous solutions studied with femtosecond broad-band transient absorption spectroscopy ${ }^{14,25,27}$

${ }^{\mathrm{h}}$ Transient absorption signals were recorded by the pump-probe technique ${ }^{29}$.
The second factor, accounting for the larger stabilization of $\mathrm{T}_{\mathrm{QM}}^{3}$ Min- $\pi \pi^{*}$ and its different decay path, call for stronger electrostatic interactions: indeed, large opposite charges exist on the ${ }^{3} \mathrm{O}_{4}$ and ${ }^{5} \mathrm{C}_{4}$ atoms $(-0.62$ and +0.72 , respectively), that lies on top of each other in the FC point (Figure 1b, $3.40 \AA$ ) and get even closer $(3.17 \AA)$, and stronger (0.73 and +0.85 , respectively), in

the excited minimum. It's important to point out that we are exclusively speaking about an exciton $\pi \pi^{*}$ state evolution into the $\mathrm{T}^{3}{ }_{\mathrm{QM}}$ monomer-like path, never involving Thy-Thy CT states which are sistemtically higher in energy along the present decay route (see CT energies in Table $\mathrm{S} 3$ for Min- $\mathrm{S}_{0}$ and $\mathrm{T}_{\mathrm{QM}}^{3}$ Min- $\pi \pi^{*}$ critical points, respectively, supported by a detailed charge distribution documentation in the SI). The $\mathrm{T}^{3}{ }_{\mathrm{QM}}$ Min- $\pi \pi^{*}$ charges on ${ }^{3} \mathrm{O}_{4}$ and ${ }^{5} \mathrm{C}_{4}$ are both significantly larger than the ones found in the other minimum $\mathrm{T}_{\mathrm{QM}}^{5}$ Min- $\pi \pi^{*}(-0.61$ and +0.62 , respectively), where they also lie more far apart (3.60 ̊̊), see Figure 1a. This behaviour is once again triggered by the ${ }^{3} \mathrm{O}_{4^{----}} \mathrm{C}_{4}$ bonding vs. antibonding character of the $\pi^{*}$ orbitals involved in the two excitations ( $\mathrm{L}$ and $\mathrm{L}+1$ for $\mathrm{T}^{3}{ }_{\mathrm{QM}}$ and $\mathrm{T}^{5}{ }_{\mathrm{QM}}$ respectively, see Figure 2a). It is thus apparent that the $\mathrm{T}_{\mathrm{QM}}^{3}$ Min- $\pi \pi^{*}$ stationary point is more stable compared to the 'pure' monomer-like Min$\pi \pi^{*}\left(\mathrm{~T}_{\mathrm{QM}}^{5}\right.$ Min- $\left.\pi \pi^{*}\right)$ also because of this additional attractive electrostatic component which acts 
in concert with the above-mentioned electronic $\pi \pi$-interaction in lowering the energy. Both these components, however, are lost when reaching the decay funnel, where the ${ }^{3} \mathrm{O}_{4}-{ }^{--}{ }^{5} \mathrm{C}_{4}$ distance is indeed enlarged and the base looses its planarity and consequently the stabilizing component due to the electronic $\pi \pi$-interaction (see the $\mathrm{T}^{3}{ }_{\mathrm{QM}} \mathrm{CI}-\pi \pi^{*} / \mathrm{S}_{0}$ structure in Figure $1 \mathrm{~b}$ and compare it to Min- $\mathrm{S}_{0}$ (shown in red). This is also the reason why the two conical intersections are roughly degenerate and, consequently, a sloped crossing is observed, leading to a significantly larger energy barrier (ca. $0.47 \mathrm{eV}$, see Figure 1b). While errors in CASPT2 energies and barriers may well fall within $\sim 0.2 \mathrm{eV}$ and vibrational levels (as well as ZPE corrections) are not accounted for in the present study, still, and quite notably, the computed barrier and energy profile may well account for longer life times in the order of tens to hundreds picoseconds $\left(\mathrm{t}_{3}\right)$ at least, also depending on the initially excited vibrational mode, that may favour or not the access to the CI$\pi \pi * / \mathrm{S}_{0}$ crossing.

Concerning the $\mathrm{T}^{5}$ and $\mathrm{T}^{3}$ puckering/methyl motion in the opposite directions $\left(\mathrm{T}_{\mathrm{QM}}^{5}\right.$ and $\left.\mathrm{T}_{\mathrm{QM}}^{3}{ }^{\prime}\right)$, the decay paths are shown in the lower part of Figure 1. Both the $\mathrm{T}_{\mathrm{QM}}^{5}$, and $\mathrm{T}^{3}{ }_{\mathrm{QM}}$ ' decay mechanisms entails a certain base-base approach (due to the ring distortion, Figure 1c and d) supported by an electronic transition to the inter-base bonding $\pi^{*}(\mathrm{~L})$ orbital (Figure $\left.2 \mathrm{a}\right)$. This causes the loss of the DNA Thy-Thy equilibrium distance (based on $\pi$-interactions), and the following localization of the excitation on a single base (more evident in $\mathrm{T}^{3}{ }_{\mathrm{QM}}$, Figure $2 \mathrm{c}, 2 \mathrm{~d}$ and Scheme S2). This leads to a 'boat-like' ring deformation minima $\left(\mathrm{T}^{5}{ }_{\mathrm{QM}}\right.$ ' and $\mathrm{T}^{3}{ }_{\mathrm{QM}}$ ' Min- $\pi \pi^{*}$, Figure 1c and d) closely resembling the free solvated nucleoside behaviour ${ }^{5}$ (see the torsion values in Table S1). The deformation coordinate clearly reduces the ${ }^{5} \mathrm{~N}_{3^{---}}{ }^{3} \mathrm{~N}_{3}$ inter-base distance, both nitrogens holding large negative charges (e.g. -0.95 and -0.96 in $\mathrm{T}_{\mathrm{QM}}^{3}$ ' Min- $\pi \pi^{*}$, see Figure 
$1 \mathrm{c}$ and d). This causes repulsive interactions, enhanced by the anti-bonding nature of the Min- $\mathrm{S}_{0}$ $\pi^{*}$ (L) orbital between the two nitrogens atoms (observable in Figure 2d), which should explain the minor $\mathrm{T}_{\mathrm{QM}}^{3}$ ' Min- $\pi \pi^{*}$ stabilization (Figure 1d). A slightly larger Min- $\pi \pi^{*}$ stabilization is observed in the equivalent $\mathrm{T}_{\mathrm{QM}}^{5}$, decay path (in spite of the ${ }^{5} \mathrm{~N}_{3^{---}}{ }^{3} \mathrm{~N}_{3}$ approach, Figure 1c), caused by two bonding electronic/electrostatic interaction: $\pi$-stacking interaction is, in part,

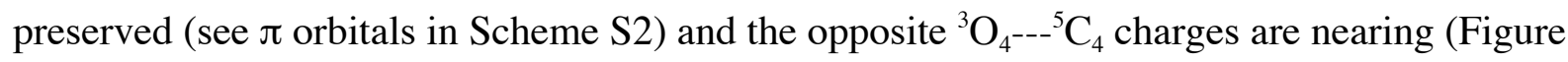
1c), even if are both significantly smaller than the ones found in the most stable $\mathrm{T}_{\mathrm{QM}}^{3}$ Min- $\pi \pi^{*}$ minimum.

In conclusion, the four $\pi \pi^{*}$ decay paths, found for a couple of QM stacked Thys inside DNA, qualitatively account for all the three life times observed in poly(A) $(\mathrm{T})$ multimers reported in Table 2 (as well as for poly $(\mathrm{T})$ ): $\tau_{1}$ and $\tau_{2}$ refer to the classical monomer-like decay also observed in solvated isolated bases (Table 2), that is here associated to the $\mathrm{T}_{\mathrm{QM}}^{5}, \mathrm{~T}_{\mathrm{QM}}^{5}$ ' and $\mathrm{T}_{\mathrm{QM}}^{3}$ ' decay routes, while $\tau_{3}$ is related to the $\mathrm{T}^{3}{ }_{\mathrm{QM}}$ path which is more affected by stabilizing electronic/electrostatic/steric interactions which modify the 'pure' monomer-like deactivation by lowering the $\pi \pi^{*}$ minimum, and consequently leading to a non-negligible barrier to reach the CI$\pi \pi^{*} / \mathrm{S}_{0}$.

Fairly enough, it should be stated that the longest life time $\left(\tau_{3}\right)$ has been also assigned in pyrimidines to $n \pi^{*}$ population and its decay ${ }^{4,12,24}$, as already mentioned in the introduction. As shown in Table 2, $\tau_{3}$ lifetimes recorded for single solvated pyrimidine nucleosides are three- to 6-fold-longer than the corresponding nucleobases, revealing an effect of ribosyl substitution on electronic energy relaxation. Notably, this effect has not been observed in purines ${ }^{12,38}$. This behaviour can be easily explained looking at the reaction coordinate that drives $n \pi^{*}-\pi \pi^{*}$ internal 


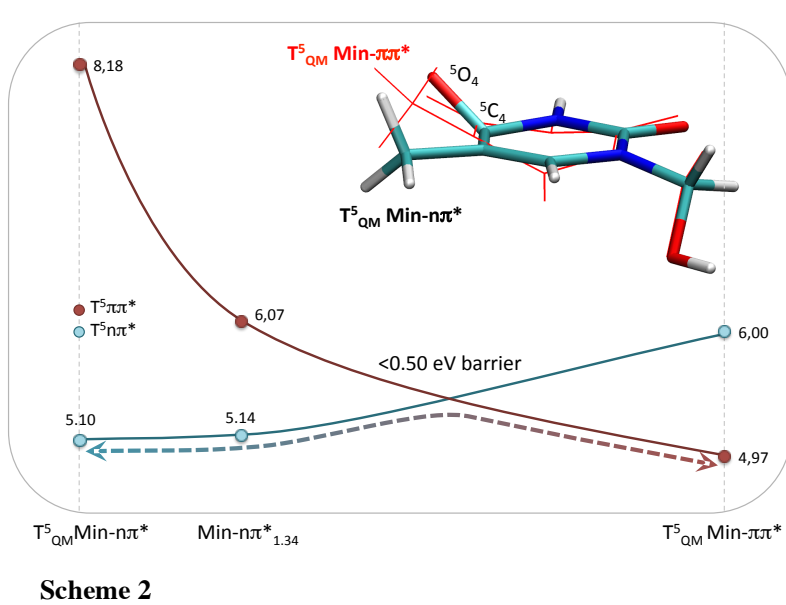

conversion (and back population) through the $\mathrm{n} \pi * / \pi \pi^{*} \mathrm{CI}$ : as recently shown ${ }^{24}, \mathrm{n} \pi^{*}$ may behave as a trap of the excited state population in solvated pyrimidine nucleosides and its decay easily occurs through repopulation of the $\pi \pi^{*}$ state $^{4,6}$, as it provides the route to the lowest energy, and more easily accessible, decay funnel. Scheme 2 represents the coordinate connecting the two minima and the overlapping structures $\mathrm{T}_{\mathrm{QM}}^{5}$ Min-n $\pi^{*}$ and Min- $\pi \pi^{*}$ (in red), showing that the population exchange involves large ring distortions and an upper limit for the barrier slightly lower than $0.5 \mathrm{eV}$ (roughly resembling solvated thymidine $\mathrm{e}^{24}$ ). Notably, these motions largely involve the $\mathrm{N}_{1}$ atom (the one bonded to the sugar moiety in pyrimidines) and its substituent as shown in Table $\mathrm{S} 1$ (see torsional angles $\mathrm{A}, \mathrm{F}, \mathrm{B}$, and $\mathrm{E}$ which all imply $\mathrm{N}_{1}$ ). These distortions are obviously very sensitive to the $\mathrm{N}_{1}-\mathrm{R}$ substitution, in particular when $\mathrm{R}=$ Hydrogen (as found in bare nucleobases) is replaced with a sugar or a sugar+phosphate, as found in nucleosides or nucleotides and DNA. A plausible hypothesis, endorsed firstly here, is that $n \pi^{*} \rightarrow \pi \pi^{*}$ repopulation is more demanding when sterically congested substituents are involved. This hindrance needs a longer time to occur that accounts for the appearance of a life time $\tau_{3}$ even longer (falling in the order of hundreds ps) than in the isolated nucleobases (Table 2). The assumption that the longer lifetime of pyrimidine nucleosides is due to $n \pi^{*}$ state trapping, is supported by the experimental negligible radiative transition rate to $\mathrm{S}_{0}$ observed for this component ${ }^{12}$. It is further confirmed by the abovementioned experimental observation that purine nucleosides/nucleotides do not display the longer lifetimes. In purines, indeed, the sugar moiety 
is bonded to the 5-membered ring, not to the photoreactive 6-membered ring: thus, this doesn't hinder the dihedral distortion and no tens or hundred ps life times are detected in AMP or GMP (see Table 2). Additionally, we have to point at the similar behaviour of the $n \pi^{*}$ states in isolated Thimidines and in stacked Thys in $\mathrm{DNA}^{24}$, respectively. We above explained the important role played by p-staking interactions in tuning the $\mathrm{T}^{3}{ }_{\mathrm{QM}}$ monomer-like path. These interactions are reduced in a state involving an $\mathrm{n}$ orbital instead of a $\pi$ orbital, thus accounting for the comparable $n \pi^{*}$ state profile found in the two different environments. In conclusion, the longer decay time $\left(\tau_{3}\right)$ observed in DNA duplexes containing stacked Thys may be well explained by both intrabase decay paths, namely i) the np* population and decay together with ii) the direct $\pi \pi^{*}$ decay, thus calling for competitive routes in accounting for the $>100$ ps decay component.

Summarizing, here we give an advanced computationally based picture of the evolution and decay of an excitonic state of two stacked Thys within DNA, fully accounting for electronic, electrostatic and steric interactions to realistically model the biological system. This is a realistic model of DNA considering that dimeric excitons dominate the photon absorption process ${ }^{33}$ either in homo- and hetero-DNA multimers that show at least two stacked homo-bases. Four different intra-base $\pi \pi^{*}$ decay routes accounts for all the possible classical 'ethene-like' mechanism, due to the asymmetric environment experienced by Thys in B-DNA ${ }^{31,35}$. Three of them roughly resemble the decay path energy profile found in isolated solvated nucleosides, showing a very flat decay path with a tiny barrier along the ring-puckering/methyl-bending reaction coordinate, and accounting for the two ultrafast $\left(\tau_{1}\right.$ and $\left.\tau_{2}\right)$ decay components observed. However, the fourth and energetically favourite path leads instead to a more stable excited state minimum, mainly due to stabilizing $\mathrm{T}^{5}-\mathrm{T}^{3}$ electronic and electrostatic interactions, which entails to overpass a 0.5 
$\mathrm{eV}$ energy barrier to reach $\pi \pi^{*} / \mathrm{S}_{0} \mathrm{CI}$, and thus accounting for the third $\left(\tau_{3}\right)$ decay component observed in the order of hundreds ps, that is typical of poly $(A) \cdot(T)($ or poly $(T))$ systems. A competitive population of the $n \pi^{*}$ state, and its decay, does also contribute to the longest lifetime signal.

\section{ASSOCIATED CONTENT}

Supporting Information. Further computational details, critical points orbitals, $\mathrm{T}_{\mathrm{QM}}^{5}$ ' and $\mathrm{T}_{\mathrm{QM}}^{3}$ ' structures, np* minima, Cartesian coordinates.

\section{AUTHOR INFORMATION}

\section{Notes}

The authors declare no competing financial interests.

\section{ACKNOWLEDGMENTS}

M.G. acknowledges support by the H2020-MSCA-ITN-2017 LightDyNAmics project (GA n. 765266)

\section{REFERENCES}

1. Crespo-Hernandez, C. E.; Cohen, B.; Hare, P. M.; Kohler, B., Ultrafast excitedstate dynamics in nucleic acids. Chem Rev 2004, 104 (4), 1977-2019.

2. Middleton, C. T.; de La Harpe, K.; Su, C.; Law, Y. K.; Crespo-Hernández, C. E.; Kohler, B., DNA Excited-State Dynamics: From Single Bases to the Double Helix. Annual Review of Physical Chemistry 2009, 60 (1).

3. Crespo-Hernández, C. E.; Cohen, B.; Kohler, B., Base stacking controls excitedstate dynamics in A·T DNA. Nature 2005, 436.

4. Improta, R.; Santoro, F.; Blancafort, L., Quantum Mechanical Studies on the Photophysics and the Photochemistry of Nucleic Acids and Nucleobases. Chemical Reviews 2016, 116 (6), 3540-3593. 
5. Pepino, A. J.; Segarra-Martí, J.; Nenov, A.; Improta, R.; Garavelli, M., Resolving Ultrafast Photoinduced Deactivations in Water-Solvated Pyrimidine Nucleosides. The Journal of Physical Chemistry Letters 2017, 8 (8), 1777-1783.

6. Gustavsson, T.; Bányász, Á.; Lazzarotto, E.; Markovitsi, D.; Scalmani, G.; Frisch, M. J.; Barone, V.; Improta, R., Singlet Excited-State Behavior of Uracil and Thymine in Aqueous Solution: A Combined Experimental and Computational Study of 11 Uracil Derivatives. Journal of the American Chemical Society 2006, 128 (2), 607-619.

7. Chen, J.; Thazhathveetil, A. K.; Lewis, F. D.; Kohler, B., Ultrafast excited-state dynamics in Hexaethyleneglycol-linked DNA homoduplexes made of A-T base pairs. J. Am. Chem. Soc. 2013, 135, 10290-10293.

8. Chatterley, A. S.; West, C. W.; Roberts, G. M.; Stavros, V. G.; Verlet, J. R. R., Mapping the ultrafast Dynamics of adenine onto its nucleotide and oligonucleotide by timeresolved photoelecron imaging. J. Phys. Chem. Lett. 2014, 5, 843-848.

9. Crespo-Hernández, C. E.; Kohler, B., Influence of secondary structure on electronic energy relaxation in adenine homopolymers. j. Phys. Chem. B. 2004, 108.

10. Cohen, B.; Crespo-Hernández, C. E.; Hare, P. M.; Kohler, B., Ultrafast Molecular Events in Chemistry and Biology. Elsevier ed.; Amsterdam, 2004.

11. Onidas, D.; Gustavsson, T.; Lazzarotto, E.; Markovitsi, D., Fluorescence of the DNA double helix (dA)20·(dT)20 studied by femtosecond spectroscopy effect of the duplex size on the properties of the excited states. J. Phys. Chem. B 2007, 111, 9644-9650.

12. Hare, P. M.; Crespo-Hernández, C. E.; Kohler, B., Internal conversion to the electronic ground state occurs via two distinct pathways for pyrimidine bases in aqueous solution. Proceedings of the National Academy of Sciences 2007, 104 (2), 435-440.

13. Huix-Rotllant, M.; Brazard, J.; Improta, R.; Burghardt, I.; Markovitsi, D., Stabilization of Mixed Frenkel-Charge Transfer Excitons Extended Across Both Strands of Guanine-Cytosine DNA Duplexes. The Journal of Physical Chemistry Letters 2015, 6 (12), 2247-2251.

14. Onidas, D.; Markovitsi, D.; Marguet, S.; Gustavsson, T., J. Phys. Chem. B 2002, 106, 11367.

15. Canuel, C.; Mons, M.; Piuzzi, F.; Tardivel, B.; Dimicoli, I.; Elhanine, M., Excited states dynamics of DNA and RNA bases: Characterization of a stepwise deactivation pathway in the gas phase. J. Chem. Phys. 2005, 122, 074316.

16. Yamazaki, S.; Taketsugu, T., Nonradiative Deactivation Mechanisms of Uracil, Thymine, and 5-Fluorouracil: A Comparative ab Initio Study. The Journal of Physical Chemistry A 2012, 116 (1), 491-503. 
17. Stojanović, L.; Bai, S.; Nagesh, J.; Izmaylov, A.; Crespo-Otero, R.; Lischka, H.; Barbatti, M., New Insights into the State Trapping of UV-Excited Thymine. Molecules 2016, 21 (11), 1603.

18. Improta, R., Photophysics and Photochemistry of Thymine Deoxy-Dinucleotide in Water: A PCM/TD-DFT Quantum Mechanical Study. The Journal of Physical Chemistry B 2012, 116 (49), 14261-14274.

19. Buchner, F.; Ritze, H.-H.; Lahl, J.; Lubcke, A., Time-resolved photoelectron spectroscopy of adenine and adenosine in aqueous solution. Physical Chemistry Chemical Physics 2013, 15 (27), 11402-11408.

20. Martinez-Fernandez, L.; Zhang, Y.; de La Harpe, K.; Beckstead, A. A.; Kohler, B.; Improta, R., Photoinduced long-lived charge transfer excited states in AT-DNA strands. Physical Chemistry Chemical Physics 2016, 18 (31), 21241-21245.

21. Zhang, Y.; de La Harpe, K.; Beckstead, A. A.; Improta, R.; Kohler, B., UVInduced Proton Transfer between DNA Strands. Journal of the American Chemical Society 2015, 137 (22), 7059-7062.

22. Vayá, I.; Brazard, J.; Huix-Rotllant, M.; Thazhathveetil, A. K.; Lewis, F. D.; Gustavsson, T.; Burghardt, I.; Improta, R.; Markovitsi, D., High-Energy Long-Lived Mixed Frenkel-Charge-Transfer Excitons: From Double Stranded (AT)n to Natural DNA. Chemistry A European Journal 2016, 22 (14), 4904-4914.

23. Conti, I.; Nenov, A.; Hofinger, S.; Flavio Altavilla, S.; Rivalta, I.; Dumont, E.; Orlandi, G.; Garavelli, M., Excited state evolution of DNA stacked adenines resolved at the CASPT2//CASSCF/Amber level: from the bright to the excimer state and back. Physical Chemistry Chemical Physics 2015, 17 (11), 7291-7302.

24. Pepino, A. J.; Segarra-Marti, J.; Nenov, A.; Rivalta, I.; Improta, R.; Garavelli, M., UV-induced long-lived decays in solvated pyrimidine nucleosides resolved at the MSCASPT2/MM level. Physical Chemistry Chemical Physics 2018, 20, 6877-6890.

25. Markovitsi, D., UV-induced DNA Damage: The Role of Electronic Excited States. Photochemistry and Photobiology 2016, 92 (1), 45-51.

26. Chatterley, A. S.; West, C. W.; G.Stavros, V.; Verlet, J. R. R., Time-resolved photoelectron imaging of the isolated deprotonated nucleotides. Chem. Sci. 2014, 5, 3963-3975.

27. Karunakaran, V .; Kleinermanns, K.; Improta, R.; Kovalenko, S. A., Photoinduced Dynamics of Guanosine Monophosphate in Water from Broad-Band Transient Absorption Spectroscopy and Quantum-Chemical Calculations. Journal of the American Chemical Society 2009, 131 (16), 5839-5850.

28. Altoè, P.; Stenta, M.; Bottoni, A.; Garavelli, M., Theor. Chem. Acc. 2007, 118, 219. 
29. Lange, A. W .; Herbert, J. M., Both Intra- and Interstrand Charge-Transfer Excited States in Aqueous B-DNA Are Present at Energies Comparable To, or Just Above, the $1 \pi \pi^{*}$ Excitonic Bright States. Journal of the American Chemical Society 2009, 131 (11), 3913-3922.

30. Demidov, V. V.; Potaman, V. N., HPLC photofingerprinting of conformational peculiarities and transitions in oligonucleotide duplexes. Nucleic Acids Research 1993, 21 (11), 2691-2696.

31. Conti, I.; Martínez-Fernández, L.; Esposito, L.; Hofinger, S.; Nenov, A.; Garavelli, M.; Improta, R., Multiple Electronic and Structural Factors Control Cyclobutane Pyrimidine Dimer and 6-4 Thymine-Thymine Photodimerization in a DNA Duplex. Chemistry - A European Journal 2017, 23 (60), 15177-15188.

32. Conti, I.; Altoè, P.; Stenta, M.; Garavelli, M.; Orlandi, G., Adenine deactivation in DNA resolved at the CASPT2//CASSCF/AMBER level. Physical Chemistry Chemical Physics 2010, 12, 5016-5023.

33. Nogueira, J. J.; Plasser, F.; Gonzalez, L., Electronic delocalization, charge transfer and hypochromism in the UV absorption spectrum of polyadenine unravelled by multiscale computations and quantitative wavefunction analysis. Chemical Science 2017, 8 (8), 5682-5691.

34. Cohen, B.; Crespo-Hernández, C. E.; Kohler, B., Strickler-Berg analysis of excited singlet state dynamics in DNA and RNA nucleosides. Faraday Discussions 2004, 127, $137-147$.

35. Szabla, R.; Kruse, H.; Stadlbauer, P.; Sponer, J.; Sobolewski, A. L., Sequential electron transfer governs the UV-induced self-repair of DNA photolesions. Chemical Science 2018, 9 (12), 3131-3140.

36. Lee, W.; Kodali, G.; Stanley, R. J.; Matsika, S., Coexistence of Different Electron-Transfer Mechanisms in the DNA Repair Process by Photolyase. Chemistry - A European Journal 2016, 22 (32), 11371-11381.

37. Rottger, K.; Marroux, H. J. B.; Bohnke, H.; Morris, D. T. J.; Voice, A. T.; Temps, F.; Roberts, G. M.; Orr-Ewing, A. J., Probing the excited state relaxation dynamics of pyrimidine nucleosides in chloroform solution. Faraday Discussions 2016, 194 (0), 683-708.

38. Ma, C.; Cheng, C. C.-W.; Chan, C. T.-L.; Chan, R. C.-T.; Kwok, W.-M., Remarkable effects of solvent and substitution on the photo-dynamics of cytosine: a femtosecond broadband time-resolved fluorescence and transient absorption study. Physical Chemistry Chemical Physics 2015, 17 (29), 19045-19057. 